\title{
ERDF Subsidies in Industrial Heritage Renewal and Urban Regeneration
}

\author{
GUO Yuchen ${ }^{1, ~ a, ~ W A N ~ L u, ~ b ~}$
}

${ }^{1}$ Tongji University, Siping Rd.1239, Shanghai, China 200092

${ }^{2}$ WAN Lu, Aedas Shanghai NO.1010, Huaihai Road, Xuhui district, Shanghai, China 200031

aaquarius0109@163.com, bonelookla@live.com

Keywords: history development, Ludwigshafen, ERDF .

Abstract. In this paper the history development of Ludwigshafen was introduced and financial support plan by EU was reviewed. EU funding in Ludwigshafen is divided into two stages. The first stage is 2001 - In 2006, focusing on proposed policies. The second stage is 2007-2013. It will be the basis of the first phase of implementation of the policy to space. Implementation of ERDF resulted in a wide range of urban regeneration policy, attracting EU Investment, and creating multiculturalism, as well as some deficiencies.

\section{Introduction}

Ludwigshafen locates in Baden - Wurttemberg and Rhine West Bank. Mannheim, which is another well-known industrial city in Germany across the river. BASF is the world's largest chemical factory bases. It is gathered by a small scale immigrants, both bustling city and quiet green space and contains history and culture. The city was born out of the unique charm of the city from intersection between the industrial civilization and modern art (Fig.1).

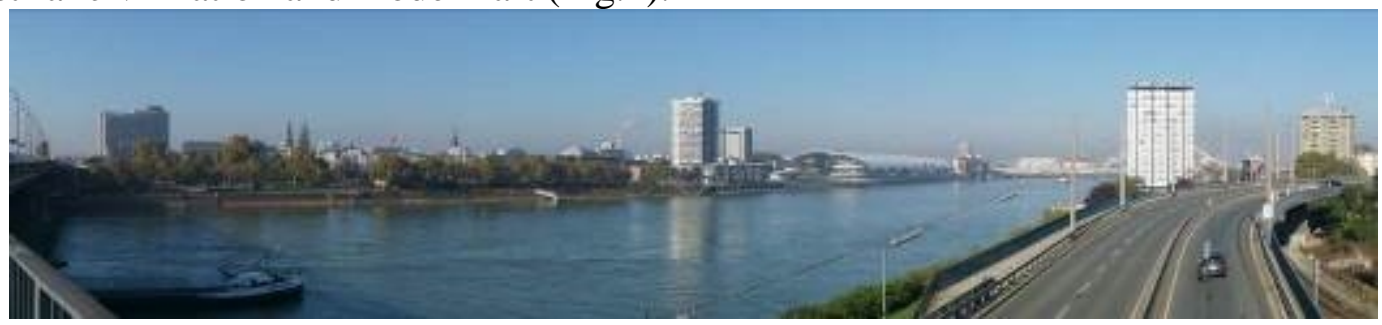

Fig.1 Ludwigshafen and Mannheim

Source: Took by Author

In 1867, Ludwigshafen rapidly growing medium-sized cities have a global vision of BASF's support in the next. Ludwigshafen has become "urban workers" status (Fig.2). After World War I and World War II, most parts of the city were destroyed, but BASF then quickly produced a huge profit, urban management departments have adequate sources of revenue to rebuild Ludwigshafen. Its population has reached an unprecedented climax, in 1970 more than 180,000 residents, beyond Mainz at the time and became one of the best urban development in Germany at that time.

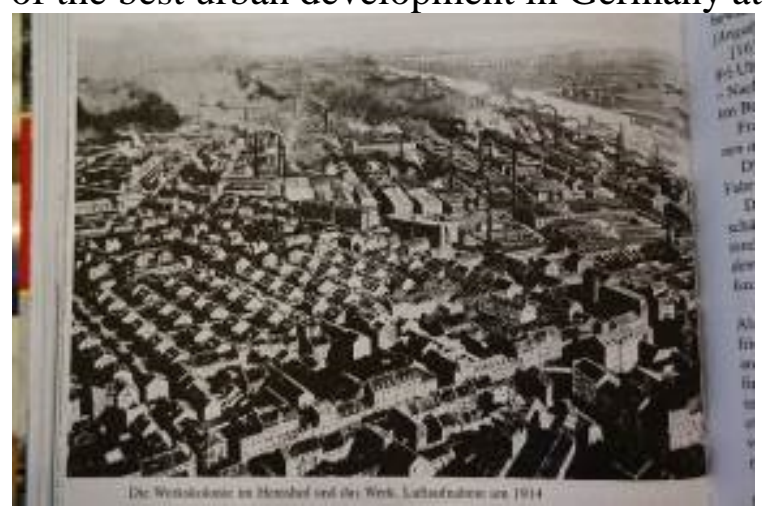

Fig.2 Ludwigshafen, 1914

Source: Took by Author in Ludwigshafen Rathaus 
From 1971, Ludwigshafen began turning a recession (Fig.3). First, in the 1960s, the brain drain and the middle class emigration to economic region brought a certain degree of decline. Second, the West German railway construction and flourish so that industry caused a huge impact on the marine Ludwigshafen increasingly weakened position as a hub of maritime trade traffic. Third, environmental pollution caused by water transport waterfront space to bring destruction. People's increasing demands for environmental quality so that the impact on the environment industry BASF has once again been pushed to public view (Fig.3).

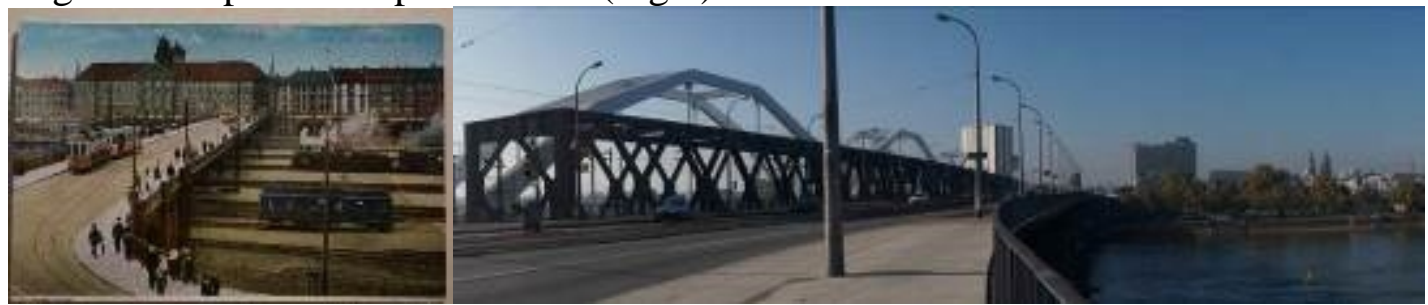

Fig.3 Ludwigshafen, historical picture (left) and Ludwigshafen, 2015 (right)

Source: Took by Author in Ludwigshafen

The new city is well developed in the industrialized German medium-sized cities (such as Ludwigshafen) are facing strong social and economic problems: radical transformation of industrial zones, mechanical and electronic decline of job opportunities, weaknesses of single industry in the local economy structure, $43.7 \%$ of foreign immigrants lack of equal opportunities, high rate of damage to the environment and so on.Although the industry has brought great success, urban industrial structure is still unbalanced urban development to produce a certain threat. How does not affect the proper adjustment of industrial structure on the basis of the development of industry itself. Then modification or replacement part of industry to conform to the development of the eras? While BASF's rapid development, the corresponding chemical industry brought serious environmental pollution. Philosopher Ernst Bloch said: "The factory dirt, forcing Ludwigshafen become a city, which is our love-hate home." How to solve the urgent environmental problems and improve the decaying urban landscape (Fig.4)?

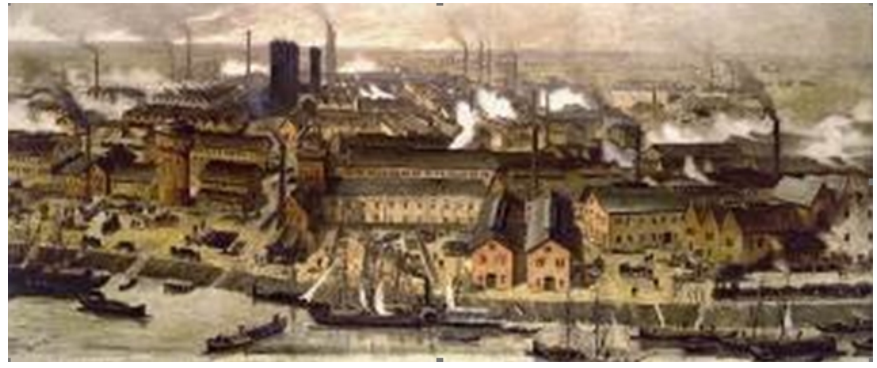

Fig.4 Air pollution in Ludwigshafen

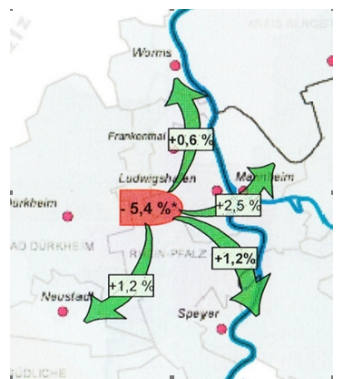

Fig.5 Local citizens are leaving

Industrial development brings the housing shortage. From1970, the number of BASF employees began to reduce and leave the city to the surrounding towns of life. In 1980--1995, for example, technical talents in Ludwigshafen decrease by 5.4\% (Fig.5). Whether we can properly sacrifice part of industrial land, building attractive urban residential areas to solve the housing problem and to solve population decline?

In the 1990s, Statistics indicated cultural facilities and recreational space in Ludwigshafen only has $2.5 \%$ of the total land use, far less than $8.3 \%$ in Mannheim and $16.7 \%$ in Heidelberg in the same period. If urban development lack of cultural base, it's hard to be resilient in the long-term development. How to make up the cultural tomography caused by rapid development?

\section{ERDF Financial Support for the Research Object}

Overview. EU funding in Ludwigshafen is divided into two stages. The first stage is 2001 - In 2006, focusing on proposed policies. The second stage is 2007-2013. It will be the basis of the first phase of implementation of the policy to space. 
Action Guide of the First Stage. The European Union will actively participate in the renewal of the cities of Mannheim and Ludwigshafen by co-financing the URBAN II Programme for Mannheim and Ludwigshafen during the 2000-2006 period. The total budget for this Programme is 21.439 million euros, of which 9.913 million euros will be provided by European funds.

The EU support to the region is not limited to in Ludwigshafen financial investment, but also to the other side of the waterfront program placed in Mannheim, make the waterfront area on both sides of the joint development of industrial heritage together to enhance urban functions. Strategic vision of the program aims to focus on three main issues to solve these problems.

First of all, economic activities and creation and preservation of jobs. New economic activity around the old train station, abandoned harbor, and the brewery former site to stimulate other jobs in addition to the industrial industry. The credits are designed to revitalize urban areas and buildings, competitive small and medium enterprises, creation of computerized economic services to employ and qualify unemployed persons. Community contributes $€ 2.8$ million to this part.

Second, measures for urban development. Projects concern reorganization of public areas, improvement of the environment and the development of transport connections. This part of the investment will focus on the construction of public space, focusing on investment in welfare facilities, health facilities, sports and cultural space. These funds can be used for local schools, day care and youth facilities. While the industrial building transformation, bring new uses for historic buildings. Also provide mutual assistance place for women, particularly female migrant families. This part has 3.6 million euros invested by the ERDF.

Socio-cultural and social integration activities. As already mentioned, in the rapid development of the industrial process, culture in Ludwigshafen appears to be insufficient. Setting up communication centers, protection of school sites and measures to reduce criminality. Bring new vitality galleries and artists working area for urban development. This part the investment by the EU is 2.9 million euros.

An additional amount of $€ 0.5$ million will finance technical assistance. This section will bring some very positive elements of urban regeneration, such as: improvement of cycling trails and sidewalks to increase the probability of interaction, improve sewage treatment systems and infrastructure, in order to transform the existing heritage of art and culture to bring new opportunity.European Union goal of this program is to "solve the urban and industrial neighborhoods recession urban crisis" and eventually attract funding from the public sector and the private sector supports about 30 million euros. After the funding of the first stage in 2001-2006, the second stage investment is based on investment income.

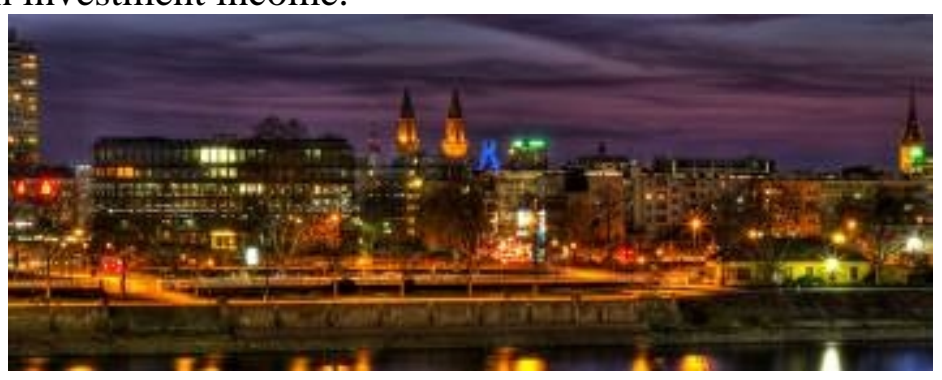

Fig. 6 Ludwigshafen waterfront

Source: http://confessedtravelholic.com/2012/05/im-moving-to-germany.html/ludwigshafen

The Implementation of the First Stage. Development concept based of Ludwigshafen "Ludwigshafen Action Plan" development strategy document, published in 2005. This document outlines the development needs of the city of Ludwigshafen on different spatial levels, and provides a new direction and focus of the transformation of regional development. The main goal of urban regeneration are urban space increase, the provision of housing, strengthening cultural life, in order to deal with various problems previously mentioned.

In residential sector, improving the existing workers' housing, and transforming the old factory office to housing, thus solving the housing problem and to reduce the vacancy rate to maximize the use of land and space. 
In the retail, dining and entertainment, the document proposes to properly compress the existing Trade and Industry, to correct the existing imbalance in the commercial and industrial status, strengthening the functions of the city retail center in northern node.

In the cultural and creative industries, policy is desirable to provide a variety of the city center, part of the abandoned plant transformation. This will create more artists' studios or theme exhibitions, art marketing space to increase the proportion of tertiary industry in cities, and with lower rents to attract young people to avoid the aging.

In the public space, we focus on building links between the old city and the waterfront and build unified public design in abandoned waterfront industrial zone. At the same time the public space may be supported and the industrial structure adjustment and improvement of urban functions. That is, a single service from the original working class turned to face diversified communication platform.

The main purpose of these renovations focused policies. They established a new way of life for the industrial city Ludwigshafen, balanced urban industrial structure and maintain the stability of the social structure.

Action Guide of the Second Stage. The second stage has total $€ 9.5$ million investment, including ERDF $€ 4.75$ million. 21 projects by ERDF in the context of a wider regeneration strategy for the city center. A clear focus (13 projects) on ERDF for regeneration of public space of physical infrastructure, of which the largest in terms of ERDF support involves renovation of the public library.

Due to the integrated approach not only structural measures to improve the urban landscape and the infrastructure are supported here, but also explicitly measures in cultural, social and educational context in order to stabilize the social structure of living in downtown population and particularly the youth improved starting opportunities to grant.

The actions financed by the ERDF are committed to the principles of sustainable development in a special way. This involves the integrated and sustainable development of economy, ecology and social aspects. Specifically, from 2007 to 2013 the following priorities to be addressed in Ludwigshafen city center in the ERDF funding:5 "socially-focused" projects are also ERDF supported: including integration for minority ethnic women, support for young parents, citizen participation and training and a competence center for senior citizens (building on URBAN II).

First, Enhancement of public space. Second, strengthening the economic development. Third, Improving infrastructure. Fourth, Education, integration and social district development. Fifth, Activation, communication (networking).

ERDF projects are clearly part of a coherent integrated strategy for the city center, with ERDF allocated to 21 of a total of 37 projects included in a single strategy.As the city center is a densely populated area, the balance between improving the physical environment, promoting entrepreneurship (mainly non-ERDF) and social measures for different target groups appears balanced.

The Implementation of the Second Stage. Different from the first phase, the second phase of policy implementation focus more on the specific space. The overall level of design strategies increase retail density of urban east-west direction and extend business functionality in northern downtown. Inner city offers modern residential, and adapt to the current development needs of housing appreciation potential, at the same time reserve living space for future creative industries (Fig.7). In addition, the strategy hope to enhance the connection by creating an attractive urban functions (Fig.7).

For the central waterfront redevelopment areas, the policy concerns of public space enhancements. The re-arrangement of new business interface will prevent blocks of vacant space and improve the living environment and enhance the quality of existing public areas (Fig.8).

As the city's waterfront backstage area, western city connect between the old town and the newer parts to each other. The plot focuses on reducing urban shortcomings and promoting the coexistence of different groups in the social structure and improving the stability and quality of life. In addition, the planning intent to transform old worker residential into office space. The rental of these offices will be very affordable, and can be used train employees. 

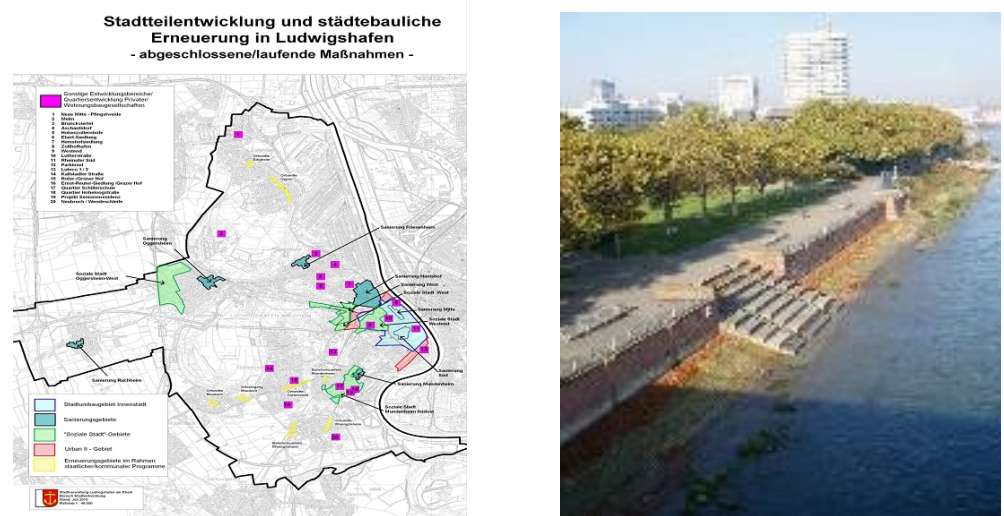

Fig.7 Ludwigshafen urban regeneration sites (left) and Ludwigshafen, 2015 (right)
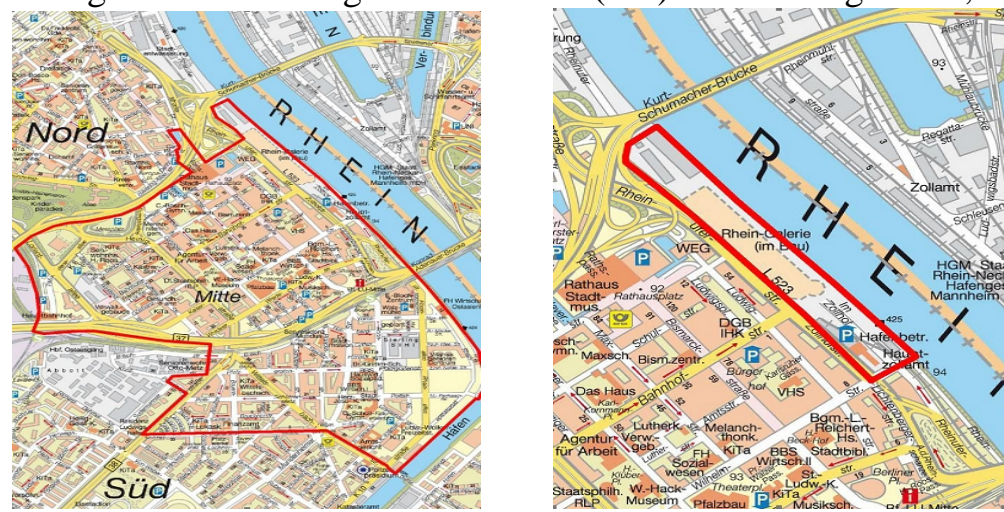

Fig.8 Ludwigshafen urban regeneration sites

Rhein-Galerie retail center, key projects in waterfront planning, which products attractive and compelling building renovation, will make a significant contribution to the revival of downtown Ludwigshafen (Fig.8). The project will create more than 1000 job opportunities, as well as good waterfront landscape and public spaces. This is an important node which shows old port transform into new urban space.

\section{Impact Assessment of ERDF}

Establishing Urban Regeneration Policy-to Implement on Specific Space. The first phase focused on proposed policy, while the second phase will be the implementation of policies and specific spatial transformation. This process is guided by the heritage renewal, resulting in a wide range of urban regeneration policy, then answer specific questions on how to achieve regeneration. In the past urban regeneration process, the majority of actions end in policy stage without the guidance of specific projects. This "from abstract to specific" process in Ludwigshafen provides a good reference.

Absorbing creative class - to improve the Urban Core Competitiveness. After Heritage renewal began, living conditions of former industrial area have been greatly improved. Landscaping and industrial elite make Ludwigshafen talents began a steady reflux and gradually above average in Germany and the Rhine-Neckar states. Talents rate grew nearly 20 percent in this decade (Fig.9). It will once again raise the economic benefits of BASF, improve Ludwigshafen's competitiveness in Germany.
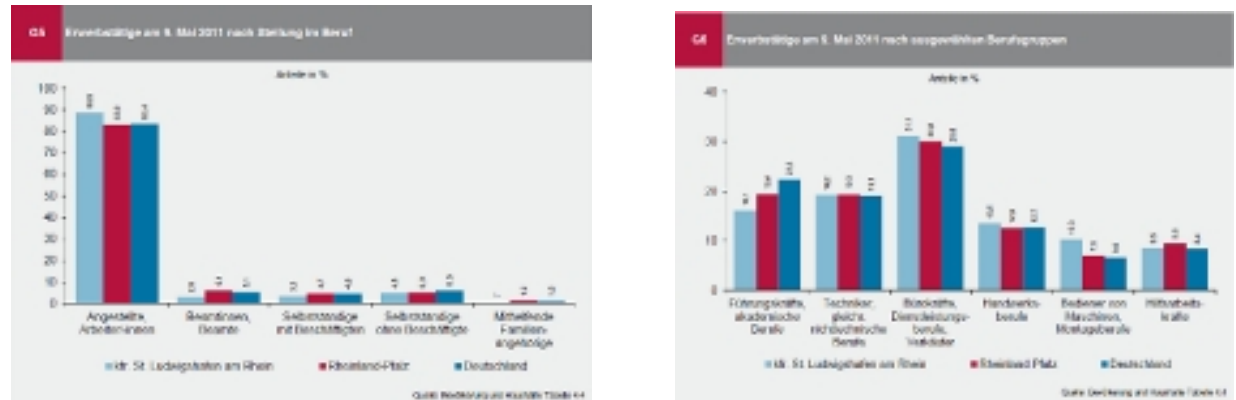

Fig.9 Ludwigshafen urban regeneration sites 
Attracting EU Investment - to Bring Increasing Economic Power. By 2013, EU investment in urban revitalization projects in Ludwigshafen accounted for only $50 \%$ of the total investment, while investment in the state accounted for $30 \%$, private investment has reached $20 \%$ of the total investment. The latter two proportion are keep increasing. The EU financial support is limited, but its impact on attracting investment in order to bring sustainable economic growth will have great help. This urban regeneration of Ludwigshafen changed from depending on external investment, gradually shifted to improve the financial situation itself.

Creating Multiculturalism - to Balance Urban Industrial Structure and Promote Cultural Quality. The transformation of the industrial heritage into cultural buildings, residential artists and exhibition space, is not only effective utilizes the building itself, but also balance the industrial structure. By considering the cultural, educational, economic and social aspects, community integration and community cohesion began to increase. Ludwigshafen is no longer simply seen as an industrial city, but also create a quality of life and urban pluralism popular culture as much as possible.

This also affected the number of new projects. In the early stages of development would not only take industry into consideration, but also will contribute to the urban cultural life. The aim is to make art and culture has the visibility, to attract new target groups and enhance the overall cultural taste.

Some Deficiencies. In the subsequent urban regeneration process, the government puts excessive investment in culture. This cause some vacancy rate in off-season. Furthermore, talent reflux and pursuit of high quality life caused a certain degree of gentrification. After 2010, the growth rate of house price improves 2 times compared with 2000-2009. It is difficult to avoid in the process of urban development. We expect Ludwigshafen can make a good response in the future.

\section{Conclusions}

In this paper the history development of Ludwigshafen was introduced and financial support plan by EU was reviewed. Implementation of ERDF resulted in Establishing Urban Regeneration Policy-to Implement on Specific Space, absorbing creative class - to improve the Urban Core Competitiveness, attracting EU Investment - to Bring Increasing Economic Power, and creating Multiculturalism - to Balance Urban Industrial Structure and Promote Cultural Quality, as well as some vacancy rate in off-season, a certain degree of gentrification and growth rate of house price.

\section{Acknowledgements}

This work was financially supported by Innovation team project of Liaoning colleges and Universities (LT2015024).

\section{References}

[1] Bähr J, Gans P. Differences in migration behaviour of Germans and Foreigners in selected cities of the federal republic of Germany[J]. Espace, populations, sociétés, 1985, 3(1): 201-214.

[2] Friedrichs J. 'Urban Revitalization Strategies and Public-Private Partnerships in German Cities’[M]. Praeger, 1998.

[3] Hospers G J. 4 Urban shrinkage in the EU[J]. Shrinking cities: a global perspective, 2014, 71: 47.

[4] Franke T, Löhr R P. Neighbourhood Management-A Key Instrument in Integrative Urban District Development[C]//EURA conference "Area-based initiatives in contemporary urban policy-innovations in city governance,'’May. 2001: 17-19. 